\title{
Longitudinal Spin Excitations and Magnetic Anisotropy in Antiferromagnetically Ordered $\mathrm{BaFe}_{2} \mathrm{As}_{2}$
}

\author{
Chong Wang, ${ }^{1}$ Rui Zhang, ${ }^{2}$ Fa Wang, ${ }^{1,3}$ Huiqian Luo, ${ }^{2}$ L. P. Regnault, ${ }^{4}$ Pengcheng Dai, ${ }^{5,2, *}$ and Yuan Li ${ }^{1,3, \dagger}$ \\ ${ }^{1}$ International Center for Quantum Materials, School of Physics, Peking University, Beijing 100871, China \\ ${ }^{2}$ Beijing National Laboratory for Condensed Matter Physics, Institute of Physics, \\ Chinese Academy of Sciences, Beijing 100190, China \\ ${ }^{3}$ Collaborative Innovation Center of Quantum Matter, Beijing 100871, China \\ ${ }^{4}$ SPSMS-MDN, UMR-E CEA/UJF-Grenoble 1, INAC, Grenoble F-38054, France \\ ${ }^{5}$ Department of Physics and Astronomy, Rice University, Houston, Texas 77005, USA
}

(Received 22 August 2013; published 30 December 2013)

\begin{abstract}
We report on a spin-polarized inelastic neutron-scattering study of spin waves in the antiferromagnetically ordered state of $\mathrm{BaFe}_{2} \mathrm{As}_{2}$. Three distinct excitation components are identified, with spins fluctuating along the $c$ axis, perpendicular to the ordering direction in the $a b$ plane and parallel to the ordering direction. While the first two "transverse" components can be described by a linear spin-wave theory with magnetic anisotropy and interlayer coupling, the third "longitudinal" component is generically incompatible with the local-moment picture. It points toward a contribution of itinerant electrons to the magnetism that is already in the parent compound of this family of Fe-based superconductors.
\end{abstract}

Among very different classes of materials, including the Fe-based superconductors (FeSCs), the cuprates, and the heavy-fermion compounds, a striking feature of unconventional superconductivity is that it commonly appears close to an antiferromagnetic (AF) phase [1]. Since magnetism may be a common thread for the pairing interaction in unconventional superconductors [2], it is important to determine the microscopic origin of the AF order. For the cuprates, it is well accepted that their Mott-insulating parent compounds have localized moments, and the spin waves can be well described by a Heisenberg model [3-5]. In the case of iron-pnictide families of FeSCs, there is no consensus on the origin of the colinear AF order in the parent compounds [6-9]. On the one hand, the parent compounds of FeSCs are semimetals with hole- and electronlike Fermi pockets at the Brillouin-zone center and zone corners, respectively [Fig. 1(a)] [10-13], and the AF order [Fig. 1(b)] may arise from nesting between the hole and electron Fermi pockets [10], much like the spindensity-wave (SDW) order in chromium [14]. On the other hand, the bad-metal phenomenology of iron pnictides [15] suggests that these materials are near a Mott transition with magnetism arising from localized moments, much like in the cuprates [16-18].

If the $\mathrm{AF}$ order in the iron pnictides arises entirely from localized moments on $\mathrm{Fe}$, spin waves from these moments should be purely transverse spin excitations (TSEs), with

\footnotetext{
*pdai@rice.edu

†yuan.li@pku.edu.cn
}

Published by the American Physical Society under the terms of the Creative Commons Attribution 3.0 License. Further distribution of this work must maintain attribution to the author(s) and the published article's title, journal citation, and DOI. moments fluctuating perpendicular to the staggered magnetization, keeping an unchanged magnitude. In contrast, if Fermi-surface nesting and itinerant electrons contribute significantly to the AF order, one would expect the presence of longitudinal spin excitations (LSEs) with fluctuating moment sizes [19-22], similar to the LSEs seen in the SDW state of chromium [23]. Although unpolarized inelastic neutron-scattering (INS) experiments have mapped out spin waves in the iron-pnictide parent compounds $\mathrm{CaFe}_{2} \mathrm{As}_{2}$ [8,24], $\mathrm{BaFe}_{2} \mathrm{As}_{2}$ [25], and $\mathrm{SrFe}_{2} \mathrm{As}_{2}$ [26], the spectra can be described by either local-moment $[8,25]$ or itinerant models $[21,24,26]$. To conclusively determine if itinerant electrons contribute to the magnetism, one needs to perform spin-polarized INS experiments to search for LSEs in the AF ordered state. In spite of considerable efforts in this direction on $\mathrm{BaFe}_{2} \mathrm{As}_{2}$ [27] and $\mathrm{NaFeAs}$ [28], no clear evidence for LSEs has been found. While the lack of LSE signal has been suggested in the previous spin-polarized INS study on $\mathrm{BaFe}_{2} \mathrm{As}_{2}$ [27], the
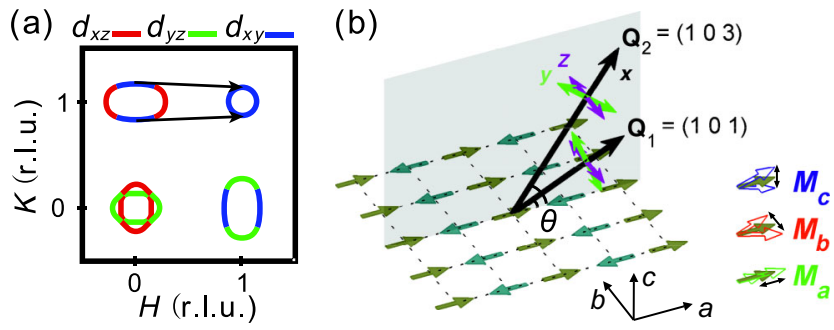

FIG. 1. (a) Fermi surface of $\mathrm{BaFe}_{2} \mathrm{As}_{2}$, reproduced from Ref. [40] using the band structure from Ref. [42]. Arrows indicate nesting vectors. (b) Spin arrangement and fluctuation directions in the $\mathrm{AF}$ phase of $\mathrm{BaFe}_{2} \mathrm{As}_{2}$. Coordinate systems for neutron polarization are indicated for two examples $\mathbf{Q}_{1}$ and $\mathbf{Q}_{2}$. 
measurements were carried out on a rather small sample (approximately $1 \mathrm{~g}$ ) with limited statistics and thus did not allow for a conclusive identification of the LSE above $20 \mathrm{meV}$.

Here, we present a spin-polarized INS study of $\mathrm{BaFe}_{2} \mathrm{As}_{2}$ in the $\mathrm{AF}$ phase, where the ordered moments are aligned along the $a$-axis direction of the orthorhombic structure [Fig. 1(b)]. By using approximately $18 \mathrm{~g}$ of coaligned crystals, we are able to overcome the statistical limitation of previous work [27] and identify three distinct spin-excitation components with magnetic moments fluctuating along the three crystallographic axes $M_{a}, M_{b}$, and $M_{c}$ [Fig. 1(b)]. The latter two TSE components can be quantitatively described by a linear spinwave model with magnetic anisotropy. The presence of the (hitherto undetected) LSE component $M_{a}$, which amounts to about $10 \%$ of the low-energy spectral weight, indicates a clear contribution from itinerant electrons. Therefore, itinerant electrons important for superconductivity also contribute to the magnetism in the parent compounds of iron pnictides.

Our high-quality $\mathrm{BaFe}_{2} \mathrm{As}_{2}$ single crystals are grown by a self-flux technique [29] and coaligned with reciprocal lattice vectors $\left(\begin{array}{lll}H & 0 & L\end{array}\right) \equiv H \mathbf{a}^{*}+L \mathbf{c}^{*}$ in the horizontal scattering plane. Here, we use the orthorhombic crystallographic notation, in which the two-dimensional AF wave vector $\left(\mathbf{Q}_{\mathrm{AF}}\right)$ corresponds to $|H|=1$ (in reciprocal lattice units, r.l.u.), and the AF zone center and boundary along $\mathbf{c}^{*}$ correspond to odd and even integer $L$ values, respectively. Our sample has an AF ordering temperature $\left(T_{N}\right)$ of about $137 \mathrm{~K}$ and a mosaic of about $1.2^{\circ}$ (Fig. S1 in Ref. [30]). The INS experiment is performed on the triple-axis spectrometer IN22 at the Institut Laue-Langevin, France. Heusler crystals are used as the spin-polarizing monochromator and analyzer, and CryoPAD is used for performing longitudinal polarization analysis. A flipping ratio of about 16 is maintained throughout our experiment. All measurements are performed in the spin-flip (SF) geometry at a temperature of $2 \mathrm{~K}$. In the $\mathrm{AF}$ ordered phase, $\mathrm{BaFe}_{2} \mathrm{As}_{2}$ forms randomly distributed orthorhombic twin domains rotated $90^{\circ}$ apart. We are effectively not sensitive to half of the sample that develops AF order at $(0 \pm 11)$ since the spin waves at $\left(\begin{array}{lll}1 & 0 L\end{array}\right)$ are well above the energy range of our measurement [25].

In the conventional coordinate system for the neutron spin polarization $(\mathbf{S}), \hat{x}$ is along the momentum transfer $(\mathbf{Q}), \hat{z}$ is vertical, and $\hat{y}$ is perpendicular to both $\hat{x}$ and $\hat{z}$ [Fig. 1(b)]. Since SF scattering probes magnetic fluctuations perpendicular to both $\mathbf{Q}$ and $\mathbf{S}$, signals that correspond to fluctuations projected along $\hat{y}\left(\sigma_{y}\right)$ and $\hat{z}\left(\sigma_{z}\right)$ can be obtained by two independent methods: $\sigma_{y}=\mathrm{SF}_{z}-$ $\mathrm{BG}=\mathrm{SF}_{x}-\mathrm{SF}_{y} \quad$ and $\quad \sigma_{z}=\mathrm{SF}_{y}-\mathrm{BG}=\mathrm{SF}_{x}-\mathrm{SF}_{z}$, where $\mathrm{SF}_{\alpha}$ denotes $\mathrm{SF}$ intensity measured with incident neutron spins along the $\alpha$ direction, and BG is background intensity. Both methods give consistent results in our study. $\sigma_{y}$ and $\sigma_{z}$ are related to the intrinsic magnetic response via

$$
\sigma_{z}=M_{b}, \quad \sigma_{y}=M_{c} \cos ^{2} \theta+M_{a} \sin ^{2} \theta,
$$

where $\theta$ is the angle between $\mathbf{Q}$ and $\mathbf{a}^{*}$ [31].

Figures 2(a) and 2(b) display raw data of energy and momentum scans at the $\mathrm{AF}$ zone center with $L=3$. The extracted $\sigma_{y}$ and $\sigma_{z}$ [Fig. 2(c)] exhibit different energy gaps, consistent with an earlier report [27]. The result of similar measurement and analysis at $L=1$ is shown in Fig. 2(d). A quantitative comparison between these measurements is presented in Fig. 2(f), where the different $\theta=$ $23.5^{\circ}$ and $52.5^{\circ}$ (for $L=1$ and 3 , respectively) determine the amount of $M_{a}$ and $M_{c}$ contributions to $\sigma_{y}$ [Eq. (1)]. The excellent agreement between the $\sigma_{z}$ data is consistent with a negligible variation in the magnetic form factor (Fig. S2 in Ref. [30]) and in the instrument resolution from $L=1$

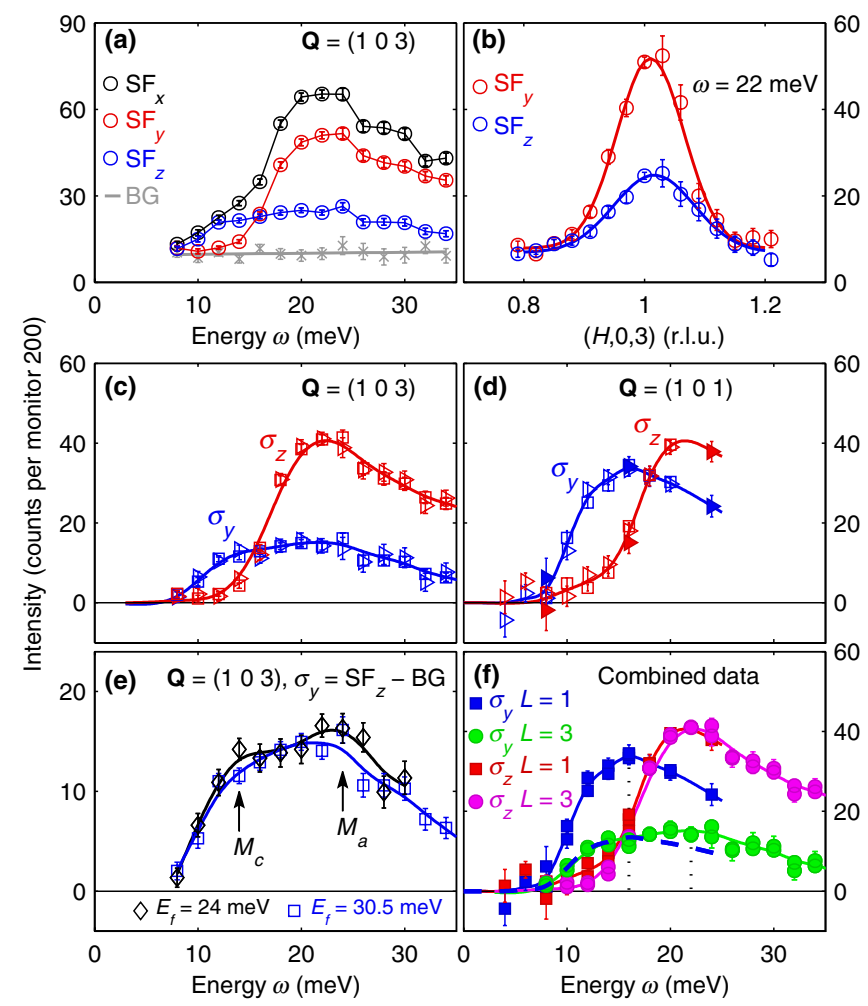

FIG. 2. (a) Energy scans at $\left(\begin{array}{lll}1 & 0 & 3\end{array}\right)$. BG is determined by fitting the $\mathrm{SF}_{y}+\mathrm{SF}_{z}-\mathrm{SF}_{x}$ intensity, which does not contain any magnetic signal, to a linear function of $\omega$. (b) $\mathbf{Q}$ scans at $\omega=$ $22 \mathrm{meV}$ fitted to a single Gaussian peak. (c),(d) Extracted $\sigma_{y}$ and

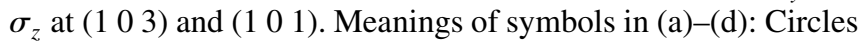
are raw data, squares are $\mathrm{SF}_{y}$ or $\mathrm{SF}_{z}$ minus $\mathrm{BG}$, triangles are $\mathrm{SF}_{x}$ minus $\mathrm{SF}_{y}$ or $\mathrm{SF}_{z}$, and empty and filled symbols are measured with final neutron energies $E_{f}=30.5$ and $50 \mathrm{meV}$, respectively. (e) Comparison of $\sigma_{y}$ data obtained with $E_{f}=24$ and $30.5 \mathrm{meV}$. (f) Combined data from (c) and (d). The dashed line indicates $\sigma_{y}$ at $\left(\begin{array}{lll}1 & 0 & 3\end{array}\right)$ after multiplying by a factor of 0.4 . Dotted lines indicate maxima of $\sigma_{y}$. Solid lines in (c)-(f) are guides to the eye. 
to $L=3$. A clear difference is found between the $\sigma_{y}$ data apart from the overall intensity change: At $L=1, \sigma_{y}$ exhibits a maximum at $16 \mathrm{meV}$, above which the signal decreases in a fashion similar to the decrease of $\sigma_{z}$ above $22 \mathrm{meV}$. At $L=3$, while $\sigma_{y}$ exhibits a rapid increase between 8 and $14 \mathrm{meV}$ similar to the behavior at $L=1$, it continues with a "plateaulike" profile to higher energies and reaches a global maximum at around $22 \mathrm{meV}$. If $\sigma_{y}$ consists of only $M_{c}$, the data for $L=1$ and $L=3$ are expected to be identical after multiplying the former by a factor of 0.44 , which accounts for the difference in $\theta$. We find the best agreement between the two data sets below $16 \mathrm{meV}$ by multiplying the $L=1$ data by 0.40 . The normalized $\sigma_{y}$ at $L=1$ [dashed line in Fig. 2(f)] lies below the $L=3$ data above $18 \mathrm{meV}$. We attribute this difference to a nonzero contribution from $M_{a}$, which, unlike $M_{c}$, is expected to increase by a factor of 4 from $L=1$ to $L=3$. To further verify this interpretation, we measure at (1 $\left.\begin{array}{lll}1 & 3\end{array}\right)$ with a smaller $E_{f}=24 \mathrm{meV}$ [Fig. 2(e)]. Indeed, the improved energy resolution (approximately $3.1 \mathrm{meV}$ at $\omega=20 \mathrm{meV}$, compared to approximately $3.9 \mathrm{meV}$ for $E_{f}=30.5 \mathrm{meV}$ ) leads to a clearer separation of the $M_{c}$ and $M_{a}$ components. These results establish the presence of an $M_{a}$ contribution to the total magnetic response at the $\mathrm{AF}$ zone center.

$\mathrm{BaFe}_{2} \mathrm{As}_{2}$ consists of FeAs layers separated by $\mathrm{Ba}$. The magnetic coupling $J_{c}$ between neighboring layers gives rise to a spin-wave dispersion along $\mathbf{c}^{*}$, with a saddle point at the AF zone boundary where $J_{c}$ can be best determined. In a recent unpolarized INS measurement at the AF zone boundary [32], a substantially smaller $J_{c}$ is found than that which was previously inferred $[8,25]$. Spin-polarized measurements have not been attempted at the AF zone boundary since this refined study. In addition to the search for LSEs, such measurements provide a stringent test of spin-wave models for describing the TSEs, with additional constraints on the model parameters.

Figure 3 displays the extracted $\sigma_{y}$ and $\sigma_{z}$ at the AF zone boundaries. For $L=2$, measurements with different $E_{f}$ are combined to satisfy the scattering kinematic constraint for the extended energy range. Additional tests (Fig. S3 in Ref. [30]) show no evidence for a distortion of data due to instrument resolution. The results are qualitatively similar to those at the $\mathrm{AF}$ zone center: (1) At $L=2$, the maximum of $\sigma_{z}$ occurs at a higher energy than $\sigma_{y}$, and both energies are higher than the corresponding values at $L=1$. (2) $\sigma_{z}$ are nearly identical at $L=2$ and $L=4$. (3) $\sigma_{y}$ reaches its maximum at a higher energy at $L=4$ than at $L=2$. From $L=2$ to $L=4$, one expects a decrease by $56 \%$ in the contribution of $M_{c}$ and an increase by $74 \%$ in the contribution of $M_{a}$. The data in Fig. 3(b) are thus consistent with maxima of $M_{c}$ and $M_{a}$ contributions at around 26 and $30 \mathrm{meV}$, respectively.

To start a quantitative discussion, we first plot in Fig. 4 the intrinsic magnetic responses at the AF zone center and

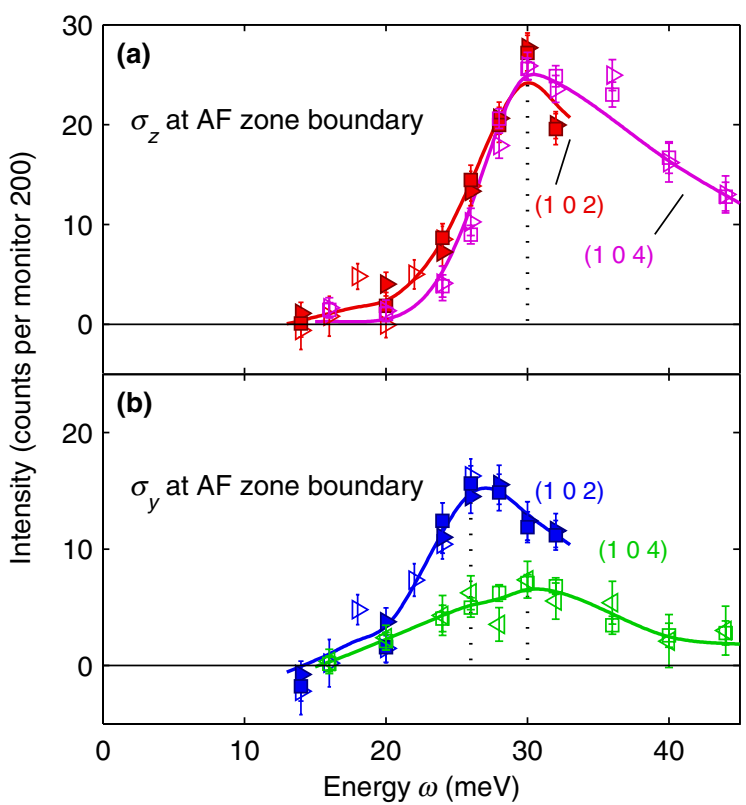

FIG. 3. (a) $\sigma_{z}$ and (b) $\sigma_{y}$ at AF zone boundaries (see Fig. S3 in Ref. [30] for the raw data). The meanings of symbols are the same as in Fig. 2. Solid lines are guides to the eye.

boundary: $M_{b}$ is determined from the average of the interpolated $\sigma_{z}$ data at $L=1,3$ and 2, 4 in Figs. 2 and 3, respectively. $M_{a}$ and $M_{c}$ are calculated from the interpolated $\sigma_{y}$ data using Eq. (1), which allows us to find a unique solution, given two measurements with different $\theta$. The energies of the TSE spin waves $\left(E_{\exp }\right.$, the energy where the signal reaches $90 \%$ of the maximum [27]) are identified from the $M_{c}$ and $M_{b}$ data and summarized in Table I. In terms of spectral weight, $M_{c}$ and $M_{b}$ are roughly equal, and

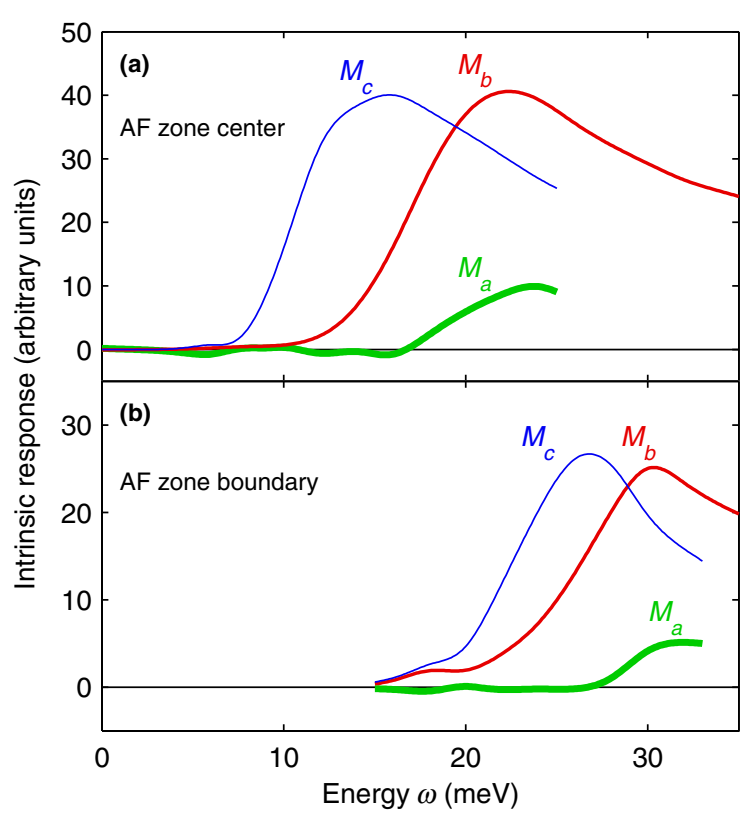

FIG. 4. Intrinsic magnetic responses (see Fig. 1 for the definition) calculated from the interpolated data in Figs. 2 and 3. 
TABLE I. Spin-wave energies calculated from Eq. (2), using $S J_{1 a}=59.2 \mathrm{meV}, S J_{2}=13.6 \mathrm{meV}, S J_{c}=0.333 \mathrm{meV}, S D_{x}=$ $0.196 \mathrm{meV}$, and $S D_{y}=-0.311 \mathrm{meV}$. The last column shows comparison with experimental data (in $\mathrm{meV}$ ). A derivation of the expressions is given in Ref. [30] (see the text and Table SI).

\begin{tabular}{lcc}
\hline \hline$M$ & $E / 2 S$ & $E_{\text {exp }} / E_{\text {cal }}$ \\
\hline$M_{b, L=1}$ & $\sqrt{\left(D_{x}-D_{y}\right)\left(4 J_{2}+2 J_{1 a}+2 J_{c}+D_{x}\right)}$ & $18.9 / 18.8$ \\
$M_{c, L=1}$ & $\sqrt{D_{x}\left(4 J_{2}+2 J_{1 a}+2 J_{c}+D_{x}-D_{y}\right)}$ & $11.6 / 11.7$ \\
$M_{b, L=2}$ & $\sqrt{\left(D_{x}-D_{y}+2 J_{c}\right)\left(4 J_{2}+2 J_{1 a}+D_{x}\right)}$ & $28.3 / 28.5$ \\
$M_{c, L=2}$ & $\sqrt{\left(D_{x}+2 J_{c}\right)\left(4 J_{2}+2 J_{1 a}+D_{x}-D_{y}\right)}$ & $24.6 / 24.4$ \\
\hline \hline
\end{tabular}

their values at the $\mathrm{AF}$ zone boundary are about $30 \%$ smaller than at the zone center. The latter observation is expected because in linear spin-wave theory, the intensity of lowenergy excitations is inversely proportional to the energy. However, as has been pointed out by Qureshi et al. [27], the equal amplitudes of $M_{c}$ and $M_{b}$, despite their energy difference, are inconsistent with the linear spin-wave theory and might indicate a necessity of resorting to more sophisticated calculations that also include itinerant electrons. Indeed, a clear LSE $M_{a}$ component is found at both the AF zone center and the zone boundary at roughly the same energies as $M_{b}$, and it amounts to about $10 \%$ of the lowenergy spectral weight. To our knowledge, our observation of a significant $M_{a}$ signal is the first direct evidence of a contribution from itinerant electrons to the spin excitations in a FeSC parent compound.

Comparing our data to the previous work by Qureshi et al. [27], the results at $L=1$ and 3 are fully consistent, but the measurement precision above $20 \mathrm{meV}$ is substantially improved in our study: The statistics are better because of the much larger sample mass, and we utilize different scattering configurations with $E_{f}=24,30.5$, and $50 \mathrm{meV}$ (Fig. 2) as further consistency checks. These experimental improvements turn out to be crucial for the detection of $M_{a}$. For comparison, we note that while the data at $\mathbf{Q}=\left(\begin{array}{lll}1 & 0 & 5\end{array}\right)$ reported in Ref. [27] are consistent with the presence of an $M_{a}$ signal at the $\mathrm{AF}$ zone center (Fig. 5), the poor statistics of the data due to small sample masses did not allow the authors to reach this conclusion. Our measurements reveal an $M_{a}$ signal at the AF zone boundary as well. Such measurements at the AF zone boundary were not attempted in Ref. [27].

We consider the following Heisenberg Hamiltonian [30] for a quantitative description of the TSE:

$$
\begin{aligned}
H= & \sum_{\mathbf{r}}\left[J_{1 a} \mathbf{S}_{\mathbf{r}} \cdot \mathbf{S}_{\mathbf{r}+\hat{x}}+J_{1 b} \mathbf{S}_{\mathbf{r}} \cdot \mathbf{S}_{\mathbf{r}+\hat{y}}\right. \\
& +J_{2}\left(\mathbf{S}_{\mathbf{r}} \cdot \mathbf{S}_{\mathbf{r}+\hat{x}+\hat{y}}+\mathbf{S}_{\mathbf{r}} \cdot \mathbf{S}_{\mathbf{r}-\hat{x}+\hat{y}}\right) \\
& \left.-D_{x}\left(S_{\mathbf{r}}^{x}\right)^{2}-D_{y}\left(S_{\mathbf{r}}^{y}\right)^{2}+J_{c} \mathbf{S}_{\mathbf{r}} \cdot \mathbf{S}_{\mathbf{r}+\hat{z}}\right],
\end{aligned}
$$

where $J_{1 a}$ and $J_{1 b}$ are the nearest-neighbor interactions along the $a$ and $b$ directions, respectively, $J_{2}$ is the

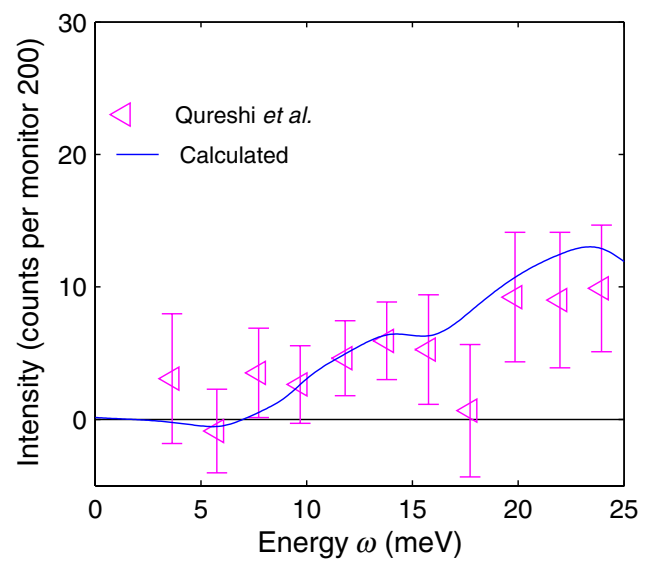

FIG. 5. $\sigma_{y}$ at $\left(\begin{array}{lll}1 & 0 & 5\end{array}\right)$ calculated from $M_{c}$ and $M_{a}$ in Fig. 4, in comparison with data reported by Qureshi et al. [27] after normalizing by the $\sigma_{z}$ values of the two experiments.

next-nearest-neighbor interaction, and $J_{c}$ is the interlayer coupling. $D_{x}$ and $D_{y}$ denote the single-ion anisotropy. In Table I, we list the expressions for the spin-wave energies. The exchange coupling parameters $J_{1 a}, J_{1 b}$, and $J_{2}$ have been determined from time-of-flight INS measurement [25]. Our data allow us to determine the remaining three parameters with four constraints. The fitted $S J_{c}$ value (Table I) is consistent with the report by Park et al. [32], taking into account the slightly different criteria of defining the spin-wave energies, and $S D_{x}$ and $S D_{y}$ are consistent with the report of Qureshi et al. [27]. Our experimental result can also be described by exchange anisotropy instead of single-ion anisotropy [30], but since the two types of anisotropy give nearly identical spin-wave dispersions, they cannot be distinguished by INS measurements.

To understand the physical origin of the LSEs, we first note that the $\mathrm{AF}$ order in $\mathrm{BaFe}_{2} \mathrm{As}_{2}$ is commensurate. This order is different from the incommensurate SDW order in chromium, and it precludes an interpretation of the LSEs as phason modes [33]. Another possible form of LSEs in itinerant antiferromagnets is the amplitude mode [19]. The lowest energy required to create such excitations occurs at the AF wave vector, consistent with our finding, and it is equal to twice the energy gap $\left(2 \Delta_{\text {SDW }}\right)$ between the magnetically split bands [19-22,34]. In the AF ordered phase, optical measurements reveal $\mathbf{q}=0$ interband transitions at 45 and $110 \mathrm{meV}$ that possibly indicate gap opening $[35,36]$, but photoemission studies show a complicated band reorganization $[12,13,37]$ without a clear gap opening at the Fermi level [13]. Since the excitations relevant to our INS data occur at finite $\mathbf{q}=\mathbf{Q}_{\mathrm{AF}}$ with $L=0$ or 1 , the correspondence between our data and $2 \Delta_{\text {SDW }}$ values inferred from optical measurements is not obvious, especially since the band structure exhibits a finite $k_{z}$ dependence [38]. The similar energies of $M_{a}$ and $M_{b}$ (Fig. 4) imply a connection between the energy scales of the itinerant and the localized electron systems. The fact that we do not observe a clear decrease of $M_{a}$ up to the 
highest energy of our measurements (Fig. 4) is consistent with the expectation that the observed $M_{a}$ is at the bottom of a LSE continuum $[20,22,34]$. Finally, we note that the energy of $M_{a}$ at the $\mathrm{AF}$ zone center is consistent with a transient optical response frequency at the verge of $\mathrm{AF}$ ordering [39].

Our result is compatible with the notion that the lowenergy spin excitations in the iron pnictides are affected by itinerant carriers, while the high-energy excitations are primarily TSEs arising from localized moments [40,41]. It would be interesting to extend the spin-polarized measurements to higher energies to determine the evolution of the LSEs. The successful description of our data by the spin-wave theory demonstrates the validity of the localmoment picture for describing the TSEs down to the lowest energy. From previous work [27], we know that the large in-plane and $c$-axis spin anisotropy disappears above $T_{N}$. In spin-polarized measurements on $\mathrm{NaFeAs}$, there is evidence for in-plane spin-excitation anisotropy in the paramagnetic orthorhombic phase [28], similar to the spin-excitation anisotropy in the tetragonal phase of superconducting $\mathrm{BaFe}_{1.904} \mathrm{Ni}_{0.096} \mathrm{As}_{2}$ [31]. It would clearly be interesting to determine how the LSE and TSE signals change above $T_{N}$ in $\mathrm{BaFe}_{2} \mathrm{As}_{2}$.

In summary, we have discovered a LSE $M_{a}$ signal and determined the TSE $M_{b}$ and $M_{c}$ components to a high precision at both the AF zone center and the zone boundary in an iron-pnictide parent compound. Since the $M_{a}$ component in nearly optimally electron-doped superconductor $\mathrm{BaFe}_{1.905} \mathrm{Ni}_{0.096} \mathrm{As}_{2}$ changes dramatically across $T_{c}$ [31], the presence of such a signal in the undoped $\mathrm{BaFe}_{2} \mathrm{As}_{2}$ suggests that itinerant electrons, which are important for superconductivity, also contribute to the magnetism in the parent compounds of iron pnictides.

We wish to thank Z.-Y. Weng, D.-X. Yao, and Y.-Z. You for stimulating discussions. Work at Peking University is supported by the NBRP of China (No. 2013CB921903) and the NSF of China (No. 11374024). Work at IOP, CAS, is supported by the NBRP of China (No. 2011CBA00110 and No. 2012CB821400) and the NSF of China (No. 11374011 and No. 91221303). H. L. and R.Z. acknowledge Project No. 2013DB03 supported by NPL, CAEP. Work at Rice University is supported by the U.S. NSF DMR-1063866 and NSF OISE-0968226.

[1] Y. J. Uemura, Superconductivity: Commonalities in Phase and Mode, Nat. Mater. 8, 253 (2009).

[2] D. J. Scalapino, A Common Thread: The Pairing Interaction for Unconventional Superconductors, Rev. Mod. Phys. 84, 1383 (2012).

[3] P. A. Lee, N. Nagaosa, and X.-G. Wen, Doping a Mott Insulator: Physics of High-Temperature Superconductivity, Rev. Mod. Phys. 78, 17 (2006).
[4] R. Coldea, S. M. Hayden, G. Aeppli, T. G. Perring, C. D. Frost, T. E. Mason, S.-W. Cheong, and Z. Fisk, Spin Waves and Electronic Interactions in $\mathrm{La}_{2} \mathrm{CuO}_{4}$, Phys. Rev. Lett. 86, 5377 (2001).

[5] N.S. Headings, S. M. Hayden, R. Coldea, and T. G. Perring, Anomalous High-Energy Spin Excitations in the High- $T_{c}$ Superconductor-Parent Antiferromagnet $\mathrm{La}_{2} \mathrm{CuO}_{4}$, Phys. Rev. Lett. 105, 247001 (2010).

[6] Q. Huang, Y. Qiu, W. Bao, M. A. Green, J. W. Lynn, Y. C. Gasparovic, T. Wu, G. Wu, and X. H. Chen, NeutronDiffraction Measurements of Magnetic Order and a Structural Transition in the Parent $\mathrm{BaFe}_{2} \mathrm{As}_{2}$ Compound of FeAs-Based High-Temperature Superconductors, Phys. Rev. Lett. 101, 257003 (2008).

[7] C. de la Cruz, Q. Huang, J. W. Lynn, J. Li, W. Ratcliff II, J. L. Zarestky, H. A. Mook, G. F. Chen, J. L. Luo, N. L. Wang, and P. Dai, Magnetic Order Close to Superconductivity in the Iron-Based Layered $\mathrm{LaO}_{1-x} \mathrm{~F}_{x} \mathrm{FeAs}$ Systems, Nature (London) 453, 899 (2008).

[8] J. Zhao, D. T. Adroja, D.-X. Yao, R. Bewley, S. Li, X.F. Wang, G. Wu, X. H. Chen, J. Hu, and P. Dai, Spin Waves and Magnetic Exchange Interactions in $\mathrm{CaFe}_{2} \mathrm{As}_{2}$, Nat. Phys. 5, 555 (2009).

[9] H. Luetkens, H.-H. Klauss, M. Kraken, F. J. Litterst, T. Dellmann, R. Klingeler, C. Hess, R. Khasanov, A. Amato, C. Baines, M. Kosmala, O. J. Schumann, M. Braden, J. Hamann-Borrero, N. Leps, A. Kondrat, G. Behr, J. Werner, and B. Büchner, The Electronic Phase Diagram of the $\mathrm{LaO}_{1-x} \mathrm{~F}_{x} \mathrm{FeAs}$ Superconductor, Nat. Mater. 8, 305 (2009).

[10] P. J. Hirschfeld, M. M. Korshunov, and I. I. Mazin, Gap Symmetry and Structure of Fe-Based Superconductors, Rep. Prog. Phys. 74, 124508 (2011).

[11] C. Liu, G. D. Samolyuk, Y. Lee, N. Ni, T. Kondo, A. F. Santander-Syro, S. L. Bud'ko, J.L. McChesney, E. Rotenberg, T. Valla, A. V. Fedorov, P. C. Canfield, B. N. Harmon, and A. Kaminski, K-Doping Dependence of the Fermi Surface of the Iron-Arsenic $\mathrm{Ba}_{1-x} \mathrm{~K}_{x} \mathrm{Fe}_{2} \mathrm{As}_{2}$ Superconductor Using Angle-Resolved Photoemission Spectroscopy, Phys. Rev. Lett. 101, 177005 (2008).

[12] L. X. Yang, Y. Zhang, H. W. Ou, J. F. Zhao, D. W. Shen, B. Zhou, J. Wei, F. Chen, M. Xu, C. He, Y. Chen, Z. D. Wang, X.F. Wang, T. Wu, G. Wu, X. H. Chen, M. Arita, K. Shimada, M. Taniguchi, Z. Y. Lu, T. Xiang, and D. L. Feng, Electronic Structure and Unusual Exchange Splitting in the Spin-Density-Wave State of the $\mathrm{BaFe}_{2} \mathrm{As}_{2}$ Parent Compound of Iron-Based Superconductors, Phys. Rev. Lett. 102, 107002 (2009).

[13] G. Liu, H. Liu, L. Zhao, W. Zhang, X. Jia, J. Meng, X. Dong, J. Zhang, G. F. Chen, G. Wang, Y. Zhou, Y. Zhu, X. Wang, Z. Xu, C. Chen, and X. J. Zhou, Band-Structure Reorganization across the Magnetic Transition in $\mathrm{BaFe}_{2} \mathrm{As}_{2}$ Seen via High-Resolution Angle-Resolved Photoemission, Phys. Rev. B 80, 134519 (2009).

[14] E. Fawcett, Spin-Density-Wave Antiferromagnetism in Chromium, Rev. Mod. Phys. 60, 209 (1988).

[15] D. N. Basov and A. V. Chubukov, Manifesto for a Higher $T_{c}$, Nat. Phys. 7, 272 (2011).

[16] Q. Si and E. Abrahams, Strong Correlations and Magnetic Frustration in the High $T_{c}$ Iron Pnictides, Phys. Rev. Lett. 101, 076401 (2008). 
[17] K. Seo, B. A. Bernevig, and J. Hu, Pairing Symmetry in a Two-Orbital Exchange Coupling Model of Oxypnictides, Phys. Rev. Lett. 101, 206404 (2008).

[18] S. Sachdev, M. A. Metlitski, and M. Punk, Antiferromagnetism in Metals: From the Cuprate Superconductors to the Heavy Fermion Materials, J. Phys. Condens. Matter 24, 294205 (2012).

[19] J. B. Sokoloff, Theory of Inelastic Neutron Scattering in the Itinerant Model Antiferromagnetic Metals. I, Phys. Rev. 185, 770 (1969); J. B. Sokoloff, Theory of Neutron Scattering in the Itinerant Model of Antiferromagnetic Metals. II, Phys. Rev. 185, 783 (1969).

[20] J. Knolle, I. Eremin, A. V. Chubukov, and R. Moessner, Theory of Itinerant Magnetic Excitations in the SpinDensity-Wave Phase of Iron-Based Superconductors, Phys. Rev. B 81, 140506(R) (2010).

[21] E. Kaneshita and T. Tohyama, Spin and Charge Dynamics Ruled by Antiferromagnetic Order in Iron Pnictide Superconductors, Phys. Rev. B 82, 094441 (2010).

[22] Y.-Z. You, F. Yang, S.-P. Kou, and Z.-Y. Weng, Magnetic and Superconducting Instabilities in a Hybrid Model of Itinerant/Localized Electrons for Iron Pnictides, Phys. Rev. B 84, 054527 (2011).

[23] S. K. Burke, W. G. Stirling, K. R. A. Ziebeck, and J. G. Booth, Magnetic Excitations in the Incommensurate Phases of Chromium Metal, Phys. Rev. Lett. 51, 494 (1983); P. Böni, B. J. Sternlieb, G. Shirane, B. Roessli, J. E. Lorenzo, and S. A. Werner, Polarization Dependence of the Magnetic Fluctuations in Cr below $T_{N}$, Phys. Rev. B 57, 1057 (1998).

[24] S. O. Diallo, V.P. Antropov, T. G. Perring, C. Broholm, J. J. Pulikkotil, N. Ni, S. L. Bud'ko, P.C. Canfield, A. Kreyssig, A. I. Goldman, and R. J. McQueeney, Itinerant Magnetic Excitations in Antiferromagnetic $\mathrm{CaFe}_{2} \mathrm{As}_{2}$, Phys. Rev. Lett. 102, 187206 (2009).

[25] L. W. Harriger, H. Q. Luo, M. S. Liu, C. Frost, J. P. Hu, M. R. Norman, and P. Dai, Nematic Spin Fluid in the Tetragonal Phase of $\mathrm{BaFe}_{2} \mathrm{As}_{2}$, Phys. Rev. B 84, 054544 (2011).

[26] R. A. Ewings, T. G. Perring, J. Gillett, S. D. Das, S. E. Sebastian, A.E. Taylor, T. Guidi, and A. T. Boothroyd, Itinerant Spin Excitations in $\mathrm{SrFe}_{2} \mathrm{As}_{2}$ Measured by Inelastic Neutron Scattering, Phys. Rev. B 83, 214519 (2011).

[27] N. Qureshi, P. Steffens, S. Wurmehl, S. Aswartham, B. Büchner, and M. Braden, Local Magnetic Anisotropy in $\mathrm{BaFe}_{2} \mathrm{As}_{2}$ : A Polarized Inelastic Neutron Scattering Study, Phys. Rev. B 86, 060410(R) (2012).

[28] Yu Song, Louis-Pierre Regnault, Chenglin Zhang, Guotai Tan, Scott V. Carr, Songxue Chi, A. D. Christianson, Tao Xiang, and Pengcheng Dai, In-Plane Spin Excitation Anisotropy in the Paramagnetic State of NaFeAs, Phys. Rev. B 88, 134512 (2013).

[29] Y. Chen, X. Lu, M. Wang, H. Luo, and S. Li, Systematic Growth of $\mathrm{BaFe}_{2-x} \mathrm{Ni}_{x} \mathrm{As}_{2}$ Large Crystals, Supercond. Sci. Technol. 24, 065004 (2011).

[30] See Supplemental Material at http://link.aps.org/ supplemental/10.1103/PhysRevX.3.041036 for more details.
[31] H. Luo, M. Wang, C. Zhang, X. Lu, L.-P. Regnault, R. Zhang, S. Li, J. Hu, and P. Dai, Spin Excitation Anisotropy as a Probe of Orbital Ordering in the Paramagnetic Tetragonal Phase of Superconducting $\mathrm{BaFe}_{1.904} \mathrm{Ni}_{0.096} \mathrm{As}_{2}$, Phys. Rev. Lett. 111, 107006 (2013).

[32] J. T. Park, G. Friemel, T. Loew, V. Hinkov, Y. Li, B. H. Min, D. L. Sun, A. Ivanov, A. Piovano, C. T. Lin, B. Keimer, Y.S. Kwon, and D.S. Inosov, Similar ZoneCenter Gaps in the Low-Energy Spin-Wave Spectra of $\mathrm{Na}_{1-\delta} \mathrm{FeAs}$ and $\mathrm{BaFe}_{2} \mathrm{As}_{2}$, Phys. Rev. B 86, 024437 (2012).

[33] R. S. Fishman and S. H. Liu, Spin Dynamics of Chromium. II. Incommensurate Alloys, Phys. Rev. B 54, 7252 (1996).

[34] R. S. Fishman and S. H. Liu, Dynamics of Commensurate Chromium Alloys, Phys. Rev. B 50, 4240 (1994).

[35] W.Z. Hu, J. Dong, G. Li, Z. Li, P. Zheng, G. F. Chen, J. L. Luo, and N. L. Wang, Origin of the Spin Density Wave Instability in $A \mathrm{Fe}_{2} \mathrm{As}_{2} \quad(A=\mathrm{Ba}, \mathrm{Sr})$ as Revealed by Optical Spectroscopy, Phys. Rev. Lett. 101, 257005 (2008).

[36] Z. G. Chen, T. Dong, R. H. Ruan, B. F. Hu, B. Cheng, W.Z. Hu, P. Zheng, Z. Fang, X. Dai, and N.L. Wang, Measurement of the c-Axis Optical Reflectance of $A \mathrm{Fe}_{2} \mathrm{As}_{2} \quad(A=\mathrm{Ba}, \mathrm{Sr})$ Single Crystals: Evidence of Different Mechanisms for the Formation of Two Energy Gaps, Phys. Rev. Lett. 105, 097003 (2010).

[37] P. Richard, K. Nakayama, T. Sato, M. Neupane, Y.-M. Xu, J. H. Bowen, G. F. Chen, J. L. Luo, N. L. Wang, X. Dai, Z. Fang, H. Ding, and T. Takahashi, Observation of Dirac Cone Electronic Dispersion in $\mathrm{BaFe}_{2} \mathrm{As}_{2}$, Phys. Rev. Lett. 104, 137001 (2010).

[38] C. Liu, T. Kondo, N. Ni, A. D. Palczewski, A. Bostwick, G. D. Samolyuk, R. Khasanov, M. Shi, E. Rotenberg, S. L. Bud'ko, P. C. Canfield, and A. Kaminski, Three- to TwoDimensional Transition of the Electronic Structure in $\mathrm{CaFe}_{2} \mathrm{As}_{2}$ : A Parent Compound for an Iron Arsenic High-Temperature Superconductor, Phys. Rev. Lett. 102, 167004 (2009).

[39] K. W. Kim, A. Pashkin, H. Schäfer, M. Beyer, M. Porer, T. Wolf, C. Bernhard, J. Demsar, R. Huber, and A. Leitenstorfer, Ultrafast Transient Generation of SpinDensity-Wave Order in the Normal State of $\mathrm{BaFe}_{2} \mathrm{As}_{2}$ Driven by Coherent Lattice Vibrations, Nat. Mater. 11, 497 (2012).

[40] P. Dai, J. Hu, and E. Dagotto, Magnetism and Its Microscopic Origin in Iron-Based High-Temperature Superconductors, Nat. Phys. 8, 709 (2012).

[41] M. Wang, C. Zhang, X. Lu, G. Tan, H. Luo, Y. Song, M. Wang, X. Zhang, E. A. Goremychkin, T. G. Perring, T. A. Maier, Z. Yin, K. Haule, G. Kotliar, and P. Dai, Doping Dependence of Spin Excitations and Its Correlations with High-Temperature Superconductivity in Iron Pnictides, Nat. Commun. 4, 2874 (2013).

[42] S. Graser, A. F. Kemper, T. A. Maier, H.-P. Cheng, P. J. Hirschfeld, and D. J. Scalapino, Spin Fluctuations and Superconductivity in a Three-Dimensional Tight-Binding Model for $\mathrm{BaFe}_{2} \mathrm{As}_{2}$, Phys. Rev. B 81, 214503 (2010). 